\title{
An Agent-based Approach to Care in Independent Living
}

\author{
Boštjan Kaluža $^{1}$, Violeta Mirchevska ${ }^{2}$, Erik Dovgan ${ }^{1}$, Mitja Luštrek $^{1}$ \\ and Matjaž Gams ${ }^{1}$ \\ ${ }^{1}$ Jožef Stefan Institute, Department of Intelligent Systems, Jamova cesta 39, \\ 1000 Ljubljana, Slovenia \\ ${ }^{2}$ Result, d.o.o., Bravničarjeva 11, 1000 Ljubljana, Slovenia \\ \{bostjan.kaluza, violeta.mircevska, erik.dovgan, \\ mitja.lustrek, matjaz.gams\} dijs.si
}

\begin{abstract}
This paper presents a multi-agent system for the care of elderly people living at home on their own, with the aim to prolong their independence. The system is composed of seven groups of agents providing a reliable, robust and flexible monitoring by sensing the user in the environment, reconstructing the position and posture to create the physical awareness of the user in the environment, reacting to critical situations, calling for help in the case of an emergency, and issuing warnings if unusual behavior is detected. The system has been tested during several on-line demonstrations.
\end{abstract}

Keywords: Multi-agent system, fall detection, disability detection, independent living, remote care.

\section{Introduction and Related Work}

The population of developed countries is aging rapidly, increasing the pressure on the working-age population to take care of the elderly. However, given the choice, most elderly people would prefer to continue to live in their own homes (aging in place) [13]. Unfortunately, the majority of elderly gradually lose their ability to function and require either additional assistance in the home or a move to an eldercare (care for the elderly) facility. Moreover, the fear of falling decreases the quality of life and increases the decline in the ability to perform daily activities.

Several systems were introduced in recent years to address some of the issues related to eldercare, e.g., fall detection. However, most of the systems developed in this context are either too expensive for mass use or of low quality. Most commercial solutions are capable only of fall detection, meaning that they recognize only a small set of hazardous situations.

Pan et al. [15], for example, presented a homecare service that tackles the problem of fall discovery using a tri-axial accelerometer and reporting such a discovery to an 
emergency center. The paper introduces a fall detector based on a neural network and a multi-agent architecture for requesting emergency services. Similar functionalities (fall detection and reporting) were also presented in other papers [2, 5, 6, 9, 18, 20] and systems [1, 19]. In addition, Isern et al. [8] reviewed several agent-based solutions for healthcare concluding that such an approach has positive affects in terms of modularity, efficiency, decentralization, flexibility, personalization, distributed planning, monitoring, pro-activity and security. In the remote care field they gave special mention to Agineru [17], a multi-agent system that monitors and processes psychological parameters on a portable device and enables physicians to access the data through a web application based on a centralized database. Besides, Koutkias et al. [10] presented a multi-agent system for the management of chronic diseases that detects anomalous cases and informs personnel; and Cervantes et al. [3] proposed a similar system to [10] for pervasive management that collects and evaluates physiological data collected by sensor agent in order to detect user's symptoms by patient's agent and to report on the situation to doctor's agents.

In the work described in this paper we aim to expand the scope of detection of hazardous situations and augment the detection system by user-adaptation. Our system presents a part of the EU FP7 project Confidence [4, 12], which main objective is to build a care system for the elderly people. An important advantage of our system is that it is based on localization hardware (that enables context-depended reasoning resulting in a lower false-alarm), which is, compared to the video-based motion-tracking systems, low-cost solution and therefore, less accurate. Nevertheless, we developed a low-cost system which accuracy is comparable to high-cost videobased systems. In addition, the localization system is especially suitable for elderly people concerned about their privacy since it is less intrusive than video-based systems. The development of the low-cost less-intrusive system is the main contribution of our work. In addition, unlike most previous work, which basically dealt with just one task, the objective of our research was twofold. The primary goal was to create a care system that is able to detect hazardous situations in short-term behavior, such as falls or a loss of consciousness, which is one of the most common tasks in eldercare. The second, rarely addressed goal was to monitor the user's behavior over longer periods of time and detect changes that indicate decreased performance. Gait disorders, for example, are an important indicator of a variety of health problems: joint and muscular diseases, neurological disorders such as Parkinson's disease etc.

The rest of the paper is organized as follows. Section 2 gives a detailed description of the architecture of our system. Section 3 shows how all the agents work together in two test scenarios: one focused on fall detection and one dealing with detection of long-term changes in behavior. Finally, Section 4 concludes the paper and outlines future work.

\section{System Architecture}

The system is based on the following six requirements. First, the system is required to monitor a person and detect an emergency situation in real time. Second, the system 
must be hardware independent, meaning that it can be easily integrated with various localization systems as the sensing component, and coupled with various schemes for user interaction as the communication component. Third, the data presentation must be increasingly more abstract, allowing an inference about the person in the environment at several abstraction levels. For example, a hazardous fall can be detected both from raw sensor data as a sudden change of vertical acceleration, and at the abstract level as a person that laid down quickly in the kitchen. Fourth, the system must allow the introduction of redundancy by combining several methods, sensors and viewpoints to solve given task. This contributes to the stability and robustness of the system as stated by the so-called principle of multiple knowledge. And finally, the system must be able to provide an insight into the person and the environment accessible to an observer.

A multi-agent architecture meets the system requirements. Each system module, task or activity is designed as an agent, providing a service. Agents are organized into groups at a specific level of abstraction and coordinated by another, hierarchically higher-level agent. Each agent can be simply modified or replaced and new or redundant agents can be easily incorporated. An agent provides a service that is requested by another agent or is triggered by some event. The data are available in the agent's output queue waiting to be used, requested or executed by another agent. Agents share their data through direct acquisition or by depositing and searching for the data in the system ontology.

With respect to the goals and requirements we present the architecture illustrated in Figure 1, which organizes the agents into seven groups. Agents from one group have the same architecture and perform logically similar functions, e.g., all sensor agents retrieve the data from sensors. The arrows indicate the communication between agents.

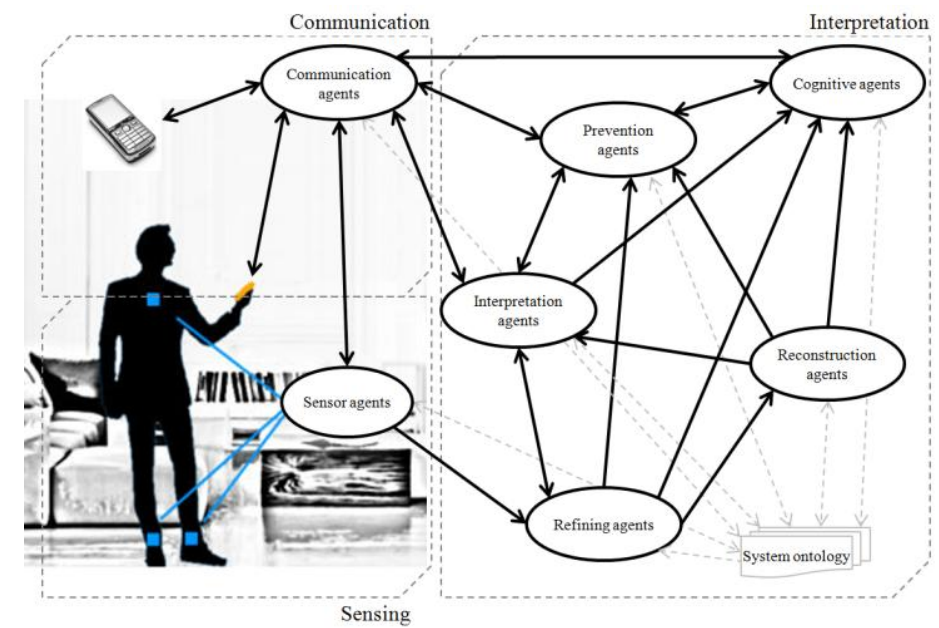

Fig. 1. The agent architecture reveals the various groups of agents. The arrows indicate the communication between agents. 
Sensor agents. An arbitrary localization hardware is employed as a sensing agent that serves raw data to the next group of agents. For example, an agent obtains the coordinates of the tag attached to the left ankle. The location agents usually report the sensor data in sequential time periods (1/10 second), but can also report other information such as "low battery".

Refining agents. The refining agents, the second agent group, represent the first abstraction layer. These agents first filter the noise using six independent methods, the most interesting of which is probably a method that corrects coordinates of body parts based on anatomic constraints. The agents then derive attributes describing the user's posture that form the foundation for the uniform representation of all the available data of the user's body.

Reconstruction agents. The reconstruction agents determine the location and posture of a person at a specific time. These agents provide essential information to activate other agents that assess the situation in the environment and accomplish the actions that are required. There are two groups of prediction agents that work in parallel: the one consisting of machine-learning agents and the one with expert-knowledge agents. The machine-learning agents compute a set of attributes (e.g., distance between tags, velocities, angles etc.) and feed them into Random Forest classifier [11]. The expertknowledge agents are designed a set of rules proclaiming and negating specific posture [14]. The outputs of both groups are merged by the meta-prediction agents based on heuristics, and learning agents that use, for example, Hidden Markov Models to predict and smooth the final classification.

Interpretation agents. The fourth group, the interpretation group of agents, tries to figure out if a situation is potentially dangerous for the user. For example, it observes that the user is lying immovable at a position where he or she is usually not lying and provides the answer to why this happened - there might be a problem, e.g., the person might have lost consciousness. This group consists of expert knowledge agents, and prediction agents based on machine-learning algorithms, while the final decision is made by a meta-prediction agent. The expert-knowledge agents contain the expert know-how related to the detection of emergency situations that may be caused by a fall or a sudden health problem. These agents are able to recognize four types of emergency situation: (1) falling detected and user lying/sitting immovable at an inappropriate place; (2) user lying/sitting immovable at an inappropriate place for long period of time; (3) falling detected and user lying/sitting at an inappropriate place for a long period of time; and (4) user lying/sitting at an inappropriate place for a very long period of time. The machine-learning agents detect emergency situations based on models induced with machine-learning techniques. Unlike the expertknowledge agents, these agents predict only the presence or absence of the emergency situation. Their reasoning is using information about the percentage of time the user was involved in specific activities detected by the reconstruction agents, as well as the percentage of time the user was immovable during given time intervals. There are two machine-learning agents, one using SVM machine-learning algorithm and the other using C4.5 algorithm. The final prediction is fused by the integration agent using heuristics. 
Prevention agents. The fifth group is the prevention-agent group. Its main task is to prevent development of a disability by monitoring how the person moves at various levels. Agents in this group observe the user's behavior; each of them collects a specific subset of the behavior data in the form of attribute vectors. They automatically build behavior models that are constantly updated using these data. Consequently, behavior models are sets of the most recent behavior attribute vectors. In particular, the agents observe the dynamics of the person's performance during the carrying out an activity, a task and even the performance during day. Since each agent only partially observes user's behavior, an integration agent collects their observations and merges them into the final behavior observation. The group consists of the following agents: (1) gait-characteristics agents are focused on a person's walk in order to recognize the change in gait using attributes such as the time the user's foot is on or off the ground, step time, step length, etc; (2) turning-characteristics agents are focused on change in direction (e.g., angular velocity, radius of the turn, speed, etc); (3) activity-characteristics agents observe the general behavior during various activities using a set of simple attributes, for example, the general speed during walking and lying, the walking speed, the time of posture transitions during sitting down and standing up, etc; and (4) spatial-activity agents are focused on the daily behavior of a person thus observing a person over a longer period of time, i.e., the dynamics of a person in the apartment by monitoring activities performed during the day combined with spatial information. Each of the prevention agents sends the messages asynchronously to the integration agent, which collects the messages for a predefined period. Afterwards, it analyses the outlier degrees of all the observed behaviors, i.e., the degrees to which they deviate from the usual behavior. If an outlier degree is higher than the high warning threshold, a warning is sent to the user.

Cognitive agents. The next group is the cognitive-state agent group: an increasingly more abstract context awareness makes it possible to construct the cognitive state of the integrated system. The system design includes the cognitive state of the user (although not implemented yet in the tested version). The cognitive layer will also include the attributes, related to the cognitive state of the user, thus constructing not only the physical, but also the cognitive state of the user, using a similar agent architecture as with the lower levels. However, these agents will use the cognitive state to perform reasoning on a wider spectrum of information with an integrated reasoning strategy.

Communication agents. The last group contains communication agents dedicated to user interaction, for example, agents that alert the person with a reply demand, make a phone call to relatives or help center, graphically display the state of the system etc. An example of the communication agent is an alarm agent that triggers when a dangerous situation is reported by other agents and shuts down when the user responds that everything is $\mathrm{OK}$. We have developed a set of graphical and communication agents: (1) an agent to inform the person about an alarm or warning; (2) an agent that interacts with the user (to raise, cancel or hold an alarm); (3) graphical agents that explain the current state of a particular agent group; (4) agents that are able to explain why a specific alarm or warning was raised. Currently, our prototype implementation runs entirely on a PC. We are in the process of extending it with advanced services and devices such as an emergency call service, a portable 
device for alarm inhibition, a mobile phone, a remote web-based monitoring application etc.

\section{Experimental results}

For the prototype deployment we have equipped a room as a near-realistic apartment of about 25 square meters. The test room contained a bed, a few chairs and tables, and was divided into six logical areas: a kitchen, where the user can prepare a meal; a sleeping area, devoted to sleeping; a living room, where the person can eat a meal, watch TV, write a letter etc.; a toilet; and a corridor, an area where the user enters and leaves the room.

Since the equipment with which the Confidence system will acquire locations is still under development, the commercially available localization system Ubisense [16] was used instead. Ubisense allows local positioning by tracking a set of reasonably small tags, which are attached to a person. A sampling frequency of around $10 \mathrm{~Hz}$ can be achieved with no more than four tags attached to a person simultaneously. Each tag maintains radio contact with a sensor mounted on the wall. These sensors use ultrawideband (UWB) technology to detect the position, and make use of both the time difference of arrival and the angle of arrival to calculate the location. In a typical open environment, a location accuracy of about $15 \mathrm{~cm}$ can be achieved across $95 \%$ of the readings [16], although it can occasionally significantly drop. Due to the fact that the Ubisense sampling rate of $10 \mathrm{~Hz}$ is limited to up to four tags, we decided to position the tags at the following locations on the body: chest, belt (optional), left and right ankle.

We have designed two sets of experiments showing the capabilities of the interpretation (fall detection) and prevention (disability detection) group of agents. Both experiments are described in the following subsections.

\subsection{Fall-detection experiments}

The first experiment explores the fall-detection capabilities of the interpretation group of agents. For this purpose, we designed a complex scenario including three situations when an alarm must be raised and three situations where a false alarm is likely. The situations in which the alarm must be raised are tripping (quick fall), fainting (slow fall, potentially not detected by accelerometers) and sliding from the chair (sliding slowly from the chair and sitting on the ground, again potentially not detected by accelerometers). The situations that might lead to a false alarm, but are actually safe, non-alarming situations, are jumping in a bed, sitting down quickly and searching under the table/bed. Both jumping in a bed and sitting down quickly contain falling, thus potentially misleading the system. For systems based on accelerometers this is the primary problem.

These two sets of situations basically reveal the ability of the interpretation-agent group to carry out context-dependent reasoning, i.e., to distinguish between an alarm situation and normal behavior consisting of the same user activities based on the context in which they are done. For example, searching under the table/bed may be falsely perceived as prolonged lying on the ground, since the person is close to the 
ground. However, it differs from lying on the ground by the amount of user movement and the length of time the user is on the ground.

The prototype was tested on recordings of five people, each performing the abovementioned test scenario five times, which is 25 recordings in total. The evaluation was performed with the leave-one-person-out methodology.

Table 1 presents the achieved accuracy of the agents in the reconstruction agent group. The first three rows represent the alarm situation (A), where the alarm must be raised, the next three rows represent false-alarm situation (FA), where alarm should not be raised, and the last row represents the overall accuracy. The columns represent the accuracy achieved by machine-learning and expert-knowledge agents, while the last column represents the results achieved by the integration agent.

Tripping and fainting were recognized quite reliably in both cases - the alarm was always triggered. What proved problematic was detecting slipping from a chair. It consists of sliding down slowly from the chair and sitting on the ground. Due to the noise in the data from the localization system, which is particularly high near the ground, sitting on the ground and sitting on a chair is sometimes difficult to distinguish. Therefore, the system is not sure what is actually the right posture or even believes that the person is moving, due to the noise.

Table 1. Performance of the reconstruction agent group when detecting alarm and non-alarm situations.

\begin{tabular}{lccc}
\hline Case $\backslash$ Accuracy [\%] & $\begin{array}{c}\text { Machine- } \\
\text { learning agents }\end{array}$ & $\begin{array}{c}\text { Expert-knowledge } \\
\text { agents }\end{array}$ & $\begin{array}{c}\text { Meta-prediction } \\
\text { agents }\end{array}$ \\
\hline A: Tripping & 40.00 & 92.00 & 100.00 \\
A: Fainting standing & 64.00 & 100.00 & 100.00 \\
A: Sliding from the chair & 32.00 & 52.00 & 52.00 \\
\hline FA: Jumping in bed & 100.00 & 96.00 & 100.00 \\
FA: Sitting down quickly & 100.00 & 100.00 & 100.00 \\
FA: Searching for & 96.00 & 88.00 & 96.00 \\
something under a table/bed & $\mathbf{7 2 . 0 0}$ & $\mathbf{8 8 . 0 0}$ & $\mathbf{9 1 . 3 3}$ \\
\hline Overall & &
\end{tabular}

The system raised only one false alarm. Difficulties were observed when searching under the bed/table. The main cause for this is the inability of the activity-recognition agents to accurately distinguish a person on all fours from a person lying on the ground.

In summary, the system performed quite well. The problems it had were rather exotic, e.g., falling from a chair and remaining sitting or being on all fours for a certain period, which is also a rather unusual situation, in particular for the elderly.

\subsection{Disability detection experiments}

The second experiment tested the ability of the prevention group of agents to detect changes in behavior, indicating present or emerging disease or illness. The testing of the general disability/disease detection would ideally require many days of recordings of daily activities. Such tests are currently in progress, but initially we condensed a full five days of activities into recordings that lasted around 25 minutes each. Two 
days of normal activities serving as a training period were recorded first, followed by a day of normal and two days of abnormal activities served as a testing period.

The agents in the prevention-agent group monitor a variety of statistics of daily living and aggregate them over specific periods. If the period over which the statistics are aggregated is shortened, we expect the condensed-day tests to be a reasonable substitution for multiple-days tests. The scenarios for the first two days represent a typical daily routine for an elderly person. There is some variation in activities, but not much in general terms since the elderly tend to follow a routine. On the fourth day the elderly person is not feeling well and as a consequence is moving slowly and rests a lot. Such behavior could occur if he/she had flu or any other general health problem. On the fifth day the elderly person is limping. As a consequence, the elderly is also moving slowly and is avoiding having to stand. The person is not lying as much as on the previous day, but is sitting more than normal.

The results of the tests are shown in Table 2. The days were divided into fiveminute observation periods (five periods each day). The periods in which each group of agents raised a warning are shaded. Despite the small number of days used in the training phase, the initial results are very promising: the prevention mechanism perceived normal behavior (white fields in Table 2) on the normal day, while on the slow and limping days, each prevention agent raised a warning at least once, often more than once and in most periods more than one agent raised a warning (gray fields in Table 2). It should be noted that the simulations were not performed by an elderly person, but by healthy persons supervised by a professional physician. The simulations were as real-life as possible for the distinctive changes in performance. This is potentially the reason for the $100 \%$ performance of the system.

Table 2. Performance of the prevention-agent group during a normal day, limping and slower movement due to a health problem. The detections are marked with gray.

\begin{tabular}{l|c|c|c|c|c}
\hline $\begin{array}{c}\text { Day part / } \\
\text { agents }\end{array}$ & $\begin{array}{c}\text { Gait } \\
\text { characterist } \\
\text { ics agents }\end{array}$ & $\begin{array}{c}\text { Turning } \\
\text { characterist } \\
\text { ics agents }\end{array}$ & $\begin{array}{c}\text { Activity } \\
\text { characterist } \\
\text { ics agents }\end{array}$ & $\begin{array}{c}\text { Spatial- } \\
\text { activity } \\
\text { agent }\end{array}$ & $\begin{array}{c}\text { Meta- } \\
\text { prediction } \\
\text { agent }\end{array}$ \\
\hline Normal 1 & & & & & \\
\hline Normal 2 & & & & & \\
\hline Normal 3 & & & & & \\
\hline Normal 4 & & & & & \\
\hline Normal 5 & & & & & \\
\hline Slow 1 & & & & & \\
\hline Slow 2 & & & & & \\
\hline Slow 3 & & & & & \\
\hline Slow 4 & & & & & \\
\hline Slow 5 & & & & & \\
\hline Limping 1 & & & & & \\
\hline Limping 2 & & & & & \\
\hline Limping 3 & & & & & \\
\hline Limping 5 & & & & & \\
\hline
\end{tabular}




\section{Conclusions and Future Work}

In this paper we have presented a multi-agent system that consists of seven groups of intelligent agents, i.e., sensor, refinement, reconstruction, interpretation, prevention, and cognitive group of agents. The agents within groups are arranged horizontally, contributing various interpretations of the situation, and vertically, providing increasingly more abstract situational awareness. Each agent in group has its own strong and weak points, while the advanced combination and integration overcomes the particular weaknesses and combines different aspects into a reliable interpretation.

The results in the fall-detection experiment show that context-dependent reasoning can interpret complex scenarios that might be misinterpreted by acceleration-based systems. In addition, the preliminary results on the disability detection are encouraging, showing a potential for the early discovery of a potential health problem that may lead to perilous conditions. Overall, hundreds of hours of tests were already performed, including an on-line presentation to EU reviewers with $100 \%$ performance both in the prepared scenarios and in the scenarios defined on-the-spot. However, the real applicability of the presented system will be demonstrated in the final year of the project, when it will be installed and tested in the homes of elderly people.

In order to increase the usability, performance and effectiveness of the multi-agent system, our work in the immediate future will focus on the following goals. The first goal is an initialization procedure that will optimize the initial parameters of the system to a particular user, for example, the user person will show how he/she usually performs some activities, specify his/her height in order to refine the agents in the reconstruction mechanism, set parameters that will describe his/her normal-day dynamics in order to relax or strengthen the alert sensitivity, set privacy-related services for reporting, monitoring, etc. Secondly, we will develop additional procedures for user adaptation during the system operation, in particular, dynamic adaptation to false alarms and undiscovered alarms. Finally, we will devise effective and pro-active agents to broaden the scope of the awareness and prevention mechanism. Ultimately, the success of the interpretative mechanism's ambitious goals depends strongly on the ability to suit and adapt to an end user.

Acknowledgments. This work was partly supported by the Slovenian Research Agency under the Research Programme P2-0209 Artificial Intelligence and Intelligent Systems, and partly from the European Community's Framework Programme FP7/2007-2013 under grant agreement No. 214986. The work of Violeta Mirchevska was partly financed by the European Union, European Social Found. We would like to thank Domen Marinčič, Rok Piltaver, Boža Cvetkovič, Blaž Strle and Damjan Kužnar for suggestions, discussions and help with the programming.

\section{References}

1. AlertOne Services Inc, iLife ${ }^{\mathrm{TM}}$ Fall Detection Sensor, http://www.falldetection.com

2. Bourke, A.K., Lyons G.M.: A threshold-based fall-detection algorithm using a bi-axial gyroscope sensor. Medical Engineering \& Physics. 30(1), 84 -- 90 (2008). 
3. Cervantes, L., Lee, Y.-S., Yang, H., Ko, S.-H., Lee, J.: Agent-based intelligent decision support for the home healthcare environment'. In: Proc. of the 1st International Conference on Advances in Hybrid Information Technology. LNAI, vol. 4413, pp. 414-424. Springer, Heidelberg (2007).

4. Confidence, http://www.confidence-eu.org

5. Doughty, K., Lewis, R., McIntosh, A. The design of a practical and reliable fall detector for community and institutional telecare. Journal of Telemedicine and Telecare. 6, 150-154 (2000).

6. Fu, Z., Culurciello, E., Lichtsteiner, P., Delbruck, T. Fall detection using an address-event temporal contrast vision sensor. In: Proceedengs of the IEEE International Symposium on Circuits and Systems, pp. 424--427 (2008).

7. Fiol-Roig, G., Arellano, D., Perales, F.J.L., Bassa, P., Zanlongo, M.: The intelligent butler: A virtual agent for disabled and elderly people assistance. In: DCAI, volume 50 of Ad-vances in Soft Computing, pp. 375--384. Springer, Heidelberg (2008).

8. Isern, D., Sanchez, D., Moreno, A.: Agents applied in health care: A review. International Journal of Medical Informatics. 79(3), 145--166 (2010).

9. Kangas, M., Konttila, A., Winblad, I., Jamsa, T.: Determination of simple thresholds for accelerometry-based parameters for fall detection. In: Proceedings of the 29th Annual International Conference of the IEEE, Engineering in Medicine and Biology Society, pp. 1367--1370 (2007)

10. Koutkias, V., Chouvarda, I., Maglaveras, N.: A multiagent system enhancing home-care health services for chronic disease management. IEEE Transactions on Information Technology in Biomedicine. 9(4), 528--537 (2005).

11. Lustrek, M., Kaluza, B.: Fall detection and activity recognition with machine learning. Informatica. 33(2), 197--204 (2009).

12. Lustrek, M., Kaluza, B., Dovgan, E., Pogorelc, E., Gams, M.: Behavior analysis based on coordinates of body tags. In: AmI '09 Proceedings of the European Conference on Ambient Intelligence. LNCS, vol. 5859, pp. 14--23. Springer, Heidelberg (2009).

13. Means, R., Richards. S., Smith, R.: Community Care: Policy and Practice. Palgrave MacMillan (2008).

14. Mirchevska, V., Lustrek, M., and Gams, M.: Combining machine learning and expert knowledge for classifying human posture. In: Proceedings of the Eighteenth International Electrotechnical and Computer Science Conference, vol. B, pp. $183--186$ (2009).

15. Pan, J.-I., Yung, C.-Y., Liang, C.-C., Lai, L.-F.: An intelligent homecare emergency service system for elder falling. In: World Congress on Medical Physics and Biomedical Engineering, pp. 424--428. Springer, (2006).

16. Steggles, P., Gschwind, S.: Ubisense - a smart space platform. Technical report, Ubisense (2005).

17. Tablado, A., Illarramendi, A., Bagues, M.I., Bermudez, J., Goni, A.: Aingeru: An innovating system for tele-assistance of elderly people. Journal on Information Technology in Healthcare. 2(3), 205--214 (2004).

18. Willis, D. J.: Ambulation Monitoring and Fall Detection System using Dynamic Belief Networks. PhD Thesis, School of Computer Science and Software Engineering, Monash University (2000).

19. Zenio: Zenio Fall Detector, http://www.zenio.be/product/8.html

20. Zhang,T., Wang, J., Liu, J., Hou, P.: Fall detection by wearable-sensor and one-class SVM algorithm. In: Lecture Notes in Control and Information Science, pp. 858--863 (2006). 